\title{
A Generalization of Dyson's Formula
}

\author{
ROLAND SÉNÉOR \\ Centre de Physique Théorique de l'Ecole Polytechnique, Paris
}

Received July 22, 1968

\begin{abstract}
We give an integral representation for tempered distributions which have more general support properties in $x$ space, than those usually assumed in the derivation of the Dyson formula.

The existence of such an integral representation is shown to be equivalent to that of a suitable extension of an analytic function: namely, given an analytic function on a section $\Omega$ of a domain of holomorphy $\Omega^{\prime}$ extend it to $\Omega$ imposing on it some growth conditions.

$L^{2}$ space methods of L. HöRMANDER are used to solve this problem of extension. In order to apply these Hilbert space techniques, it was necessary to prove two important theorems on the growth of analytic function.

From the physical point of view the formula we obtained is an integral representation for the commutator of two quasi local fields.
\end{abstract}

\section{Introduction}

Let us consider the Fourier transform of the commutator of two operators in the Haag Araki theory:

$$
f(p)=\int e^{i(p, x)}\left\langle\Omega\left|\left[A\left(\frac{x}{2}\right), B\left(-\frac{x}{2}\right)\right]\right| \Omega\right\rangle d^{4} x .
$$

In the above formula, $A$ and $B$ are local observables contained respectively in the von Neuman algebras $R\left(\mathscr{B}_{A}\right)$ and $R\left(\mathscr{B}_{B}\right)$.

$$
\begin{aligned}
& A(x)=U(x) A U(-x) \\
& B(x)=U(x) B U(-x) \\
& U(x)=e^{i(P, x)},
\end{aligned}
$$

$P$ is the energy momentum operator [4], the scalar product $(p, x)$ means $p_{0} x_{0}-p_{1} x_{1}-p_{2} x_{2}-p_{3} x_{3}$, and $\Omega$ is the vacuum state.

The tempered distribution $f$ has the following properties:

a) its support is contained outside a region $\mathscr{R}$ which is roughly bounded by two spacelike surface (for a precise description look at Refs. $[0,1,2]$ and especially pages $323-325$ of Ref. [5]).

b) According to the causality condition, its Fourier transform vanishes on the $\operatorname{set}^{1}$ :

$$
\left\{x \in \mathbb{R}_{4} \mid \frac{x}{2}+\mathscr{B}_{A} \sim-\frac{x}{2}+\mathscr{B}_{B}\right\} .
$$

${ }^{1}$ Let $S$ and $T$ be sets in $\mathbb{R}_{n}$, then $S+T=\left\{x \in \mathbb{R}_{n} \mid x=s+t ; s \in S, t \in T\right\}$; for any $x, y \in \mathbb{R}_{4}$

$x \sim y$ means $\quad(x-y)^{2}=\left(x_{0}-y_{0}\right)^{2}-\left(x_{1}-y_{1}\right)^{2}-\left(x_{2}-y_{2}\right)^{2}-\left(x_{3}-y_{3}\right)^{2}<0$. 
Conditions a) and b) are close to those encountered in the Jost-LehmanDyson representation $[1,2]$. On the latter case, indeed, condition a) does not change and condition b) simplifies (one asks for the nullity outside the light cone).

Our purpose is to extend the Jost-Lehman-Dyson representation to tempered distributions which have properties similar to a) and b).

First we modify slightly condition b). Since $A$ and $B$ are local observables $\mathscr{B}_{B}-\mathscr{B}_{A}$ is a bound set in $\mathbb{R}_{4}$.

$$
\begin{aligned}
& \text { Define }{ }^{2} \text { then } W(\alpha, \beta)=\overline{\left\{x \in \mathbb{R}_{4} \mid x \in V_{\alpha}^{-} \cap V_{\beta}^{+}\right\}} \quad \text { for } \quad \alpha>\beta \\
& D(\alpha, \beta)=\overline{\left\{x \in \mathbb{R}_{4} \mid x \in V_{\alpha}^{-} \cup V_{\beta}^{+}\right\}} \quad \text { for } \quad \alpha>\beta \\
& \alpha, \beta \in \mathbb{R}_{4} \text {, and } K_{C}=\underset{\substack{\varrho, \sigma \in K \\
p>\sigma}}{\bigcup} W(\varrho, \sigma)[5] \text {. }
\end{aligned}
$$

Then, the boundedness of $K$ implies the boundedness of $K_{C}$ and if $x$ is space-like relative to $K$ it is also space-like relative to $K_{C}$. Therefore we can take $K=K_{C}$ without altering the causality condition.

$K$ being bounded, there always exists $\alpha, \beta \in \mathbb{R}_{4}, \alpha>\beta$, such that $K$ is contained in $W(\alpha, \beta)$ and consequently condition b) implies that the support of the Fourier transform is contained in $D(\alpha, \beta)$. However we will replace $D(\alpha, \beta)$ by $D(\alpha,-\alpha)=D(\alpha)$ with $\alpha=(\alpha, 0,0,0)$. Such a domain can be obtained by a suitable change ${ }^{3}$ of coordinates in $x$-space.

Secondly, because of technical reasons (Bros' theorem) we have to restrict condition a). The region $\mathscr{R}$ has to be symmetrical with respect to a hyperplane $p_{0}=C^{t}$. Henceforth from a given $\mathscr{R}$ region we will build the largest $\mathscr{R}_{S}\left(\mathscr{R}\right.$ region symmetric with respect to $\left.p_{0}=C^{t}\right)$ region contained in $\mathscr{R}$. Notice that we can always take $p_{0}=0$ by a suitable translation in $p$ space which does not change the $x$-space support properties.

Under these assumptions, we shall prove the following:

Main Theorem. Tempered distributions satisfying the conditions

$\left.a^{\prime}\right)$ the support is contained outside a region $\mathscr{R}_{S}$,

$\left.b^{\prime}\right)$ the support of the Fourier transform is contained in $D(\alpha)$ can be written:

with

$$
f(x)=\int_{C(\alpha)} \widetilde{F}(x-t ; \boldsymbol{t}) d^{3} t
$$

i) $C(\alpha)=\left\{\boldsymbol{t} \in \mathbb{R}_{3} \mid\|\boldsymbol{t}\|=\sqrt{t_{1}^{2}+t_{2}^{2}+t_{3}^{2}} \leqq \alpha\right\} ; t=(0, \boldsymbol{t})$.

ii) $\widetilde{F}(x ; \boldsymbol{t})$ belongs to $\mathscr{S}^{\prime}\left(\mathbb{R}_{4} \times \mathbb{R}_{3}\right)$.

iii) $\widetilde{F}(x ; \boldsymbol{t})$ is, for "fuxed t", a solution of the usual Dyson's problem $\tilde{F}(x, \boldsymbol{t})=\int \Delta(x ; \sigma) \psi(x, \sigma ; \boldsymbol{t}) d \sigma, \quad \sigma \in \mathbb{R}^{+}$

${ }^{2} \alpha>\beta$ means $(\alpha-\beta)^{2}>0$ and $\alpha_{0}>\beta_{0}$

$$
V_{\alpha}^{-}=\left\{x \in \mathbb{R}_{4} \mid x<\alpha\right\} \quad V_{\beta}^{+}=\left\{x \in \mathbb{R}_{4} \mid x>\beta\right\} .
$$

3 This change does not affect the region $\mathscr{R}$ whose border is described in terms of invariants. 
$\psi(x, \sigma ; \boldsymbol{t})$ has compact support in $\boldsymbol{t}$ and is such that

$$
\int e^{i(u, x)} \psi(x, \sigma ; \boldsymbol{t}) d^{4} x=0
$$

if there exists $p \in \mathscr{R}_{S}$ such that $(p-u)^{2}=\sigma$.

iv) $\operatorname{supp}_{t} \widetilde{F}(x ; \boldsymbol{t}) \subset C(\alpha)$.

Equality (1) has to be understood as follow:

Let $\varphi(x) \in \mathscr{S}\left(\mathbb{R}_{4}\right)$ then $[3]$

$$
\langle\varphi(x), \tilde{f}(x)\rangle=\langle\varphi(y+t), \tilde{F}(y ; \boldsymbol{t})\rangle .
$$

Denote by $\varepsilon$ the map $(x, \boldsymbol{t}) \rightarrow(x, t)$. Because of condition iv) we have a mapping

$$
\varphi^{\prime}=\varphi_{0} \varepsilon: \mathbb{R}_{4} \times \mathbb{R}_{3} \rightarrow \mathscr{S}\left(\mathbb{R}_{4}\right) .
$$

Therefore there exists $\psi(y, \boldsymbol{t}) \in \mathscr{S}\left(\mathbb{R}_{4} \times \mathbb{R}_{3}\right)$ which coincides with $\varphi(y+t)$ on the support of $\widetilde{F}$; this gives a meaning to formula $\left(1^{\prime}\right)$.

Notice that iii) means the "support in $x$ " of $\tilde{F}(x ; \boldsymbol{t})$ is contained in $D(0)$.

In order to prove the usual representation, we can use two kinds of approaches. One method consists in using a differential equation $[0,1,5]$, an other one is related to the construction of an envelope of holomorphy [2].

It seems very difficult to use again the differential equations for more general support conditions in $x$-space. On the other hand, we will show that the validity of the integral representation ( 1 ) is still connected with the resolution of a problem of functions of several complex variables. More precisely, given an analytic function $f$ on a linear section of a holomorphy domain we will have to find an extension of this function to the whole space. This extension has, according to the support conditions and the temperedness, to satisfy some bounds.

In order to solve this problem we will use the $L^{2}$ space methods developed by L. HörMANDER [8], which will permit us to obtain existence theorem for analytic functions, with growth conditions at the boundary, in pseudoconvex domain.

Therefore we will first show that the support properties for $f$ are equivalent to the existence of an analytic function in the envelope of holomorphy $\Omega$ of a certain domain $\omega$.

From the general requirements of field theory, this analytic function must possess in $\omega$ some growth properties that we will extend in the second part to $\Omega$. At this stage, however, we will be confronted with the difficult question of how growth conditions at the boundary change for an analytic function when we continue it to the enveloppe of holomorphy.

In the third part, the validity of formula (1) is then shown to be equivalent to

1. the existence of a function $F$ analytic in a domain which contains $\Omega$ as a linear section and which restricts to $f$ on $\Omega$; 
2. the fulfillment by $F$ of some growth conditions.

In the last part, we will apply Hörmander's technique to solve the problem defined by 1 . and 2 .

\section{The Domains $\omega$ and $\boldsymbol{\Omega}$ Associated with $\boldsymbol{f}$ and Growth Properties in $\omega$}

The support of $f$ is contained in a domain $D(\alpha)$ which is regular [3]. We can therefore decompose $\tilde{f}$ in two tempered distributions $\tilde{f}^{+}$and $\tilde{f}^{-}$ such that

1. $\tilde{f}=\tilde{f}^{+}-\tilde{f}^{-}$.

2. $\operatorname{supp} \tilde{f}^{+} \subset\left\{\overline{x \in \mathbb{R}_{4} \mid x \in V_{-\alpha}^{+} \quad x_{0}>0}\right\}$,

$$
\text { supp } \tilde{f}^{-} \subset\left\{\overline{x \in \mathbb{R}_{4} \mid x \in V_{\%}^{-} \quad x_{0}<0}\right\} \text {. }
$$

Consider now $e^{i(p, x)}$. If $\operatorname{Im} p \in V^{+}$, that is to say if $p \in T^{+}$, the restriction of $e^{i(p, x)}$ on $\bar{V}+($ in $x)$ coincides with an element of $\mathscr{S}\left(\mathbb{R}_{4}\right)$. The action of $f^{+}$on $e^{i(p, x)}$ is well defined and the result depends analytically on $p$.

Therefore the Fourier-Laplace transforms $f^{ \pm}(p)=\left\langle\tilde{f} \pm(x), e^{i(p, x)}\right\rangle$ are analytic functions respectively in $T^{+}$and $T^{-}$. Note by b.v. $f^{+}\left(\right.$b.v. $\left.f^{-}\right)$ the tempered distribution boundary value of $f^{+}(p)\left(f^{-}(p)\right)$ when $\operatorname{Im} p$ goes to zero from inside $V^{+}\left(V^{-}\right)$. As a classical result in distribution theory we have

$$
\text { b.v. } f^{+}-\text {b.v. } f^{-}=\mathscr{F} \tilde{f}
$$

where by $\mathscr{F} \tilde{f}$ we means the Fourier transform of the tempered distribution $\tilde{f}$.

From the support condition for the Fourier transform one gets that for any $\varphi(p) \in \mathscr{S}\left(\mathbb{R}_{4}\right)$, with $\operatorname{supp} \varphi(p) \subset \mathscr{R}$

$$
\langle\mathscr{F} \tilde{f}, \varphi\rangle=0 \text {. }
$$

This result implies that for any such $\varphi$

$$
\left\langle\text { b.v. } f^{+}, \varphi\right\rangle=\left\langle\text { b.v. } f^{-}, \varphi\right\rangle \text {. }
$$

We are in position to apply the "Edge of the Wedge" theorem $[5,7]$, which states that there exists a function $f(p)$, analytic in the envelope of holomorphy $H\left(\tilde{\mathscr{R}} ; T^{+}, T^{-}\right)=\Omega$ of $\omega=\widetilde{\mathscr{R}} \cup T^{+} \cup T^{-}$with $\tilde{\mathscr{R}}$ an arbitrary small neighborhood of $\mathscr{R}$ in $\mathbb{C}_{4}$, such that

$$
\begin{array}{ll}
f(p)=f^{+}(p), & p \in T^{+} \\
f(p)=f^{-}(p), & p \in T^{-}
\end{array}
$$

and the boundary values of $f(p)$ on the reals from the tubes $T^{+}$and $T^{-}$ are respectively b.v. $f^{+}$and b.v. $f^{-}$. 
We now propose to give the growth properties of $f(p)$ in $\omega$. Since $\tilde{f}=\tilde{f}^{+}-\tilde{f}^{-}$we only need to know the properties of the Laplace transform of a tempered distribution which support is contained in the light cone.

We present here a result which is due to GLASER [7].

Lemma. Let $\tilde{f}^{+}$be a tempered distribution with support in $\bar{V}^{+}$; then there exists an integer $N>0$ and a continuous function $G$ with support in $\bar{V}+$ and of polynomial growth such that $f=\square^{N} G$.

Proof. Define

$$
\begin{aligned}
& \widetilde{F}_{0}(x)=\frac{1}{8 \pi} \theta\left(x^{2}\right) \theta\left(x_{0}\right) \\
& \widetilde{F}_{k}(x)=\frac{1}{4^{k} k !(k+1) !}\left(x^{2}\right)^{k} \widetilde{F}_{0}(x), \quad(k \geqq 1, \text { integer })
\end{aligned}
$$

then for $k \geqq \mathrm{~J}$, in the sense of distributions,

and

$$
\square \widetilde{F}_{k}(x)=\widetilde{F}_{k-1}(x)
$$

$$
\square \widetilde{F}_{0}(x)=\Delta_{R}(x ; 0), \quad \square \Delta_{R}(x ; 0)=\delta(x) .
$$

Since $\tilde{f}^{+}$is a tempered distribution over $\mathbb{R}_{4}$ with support in $\bar{V}^{+}$, there exists an integer $N^{\prime}$ and a constant $C^{\prime}>0$, such that for any $\varphi \in \mathscr{S}\left(\mathbb{R}_{4}\right)$

$$
\left|\left\langle f^{+}, \varphi\right\rangle\right|<C\|\varphi\|_{N^{\prime}} .
$$

Therefore $\tilde{f}^{+}$can be extended into a continuous linear functional on the Banach space obtained by completion of $\mathscr{S}\left(\mathbb{R}_{4}\right)$ according to the norm \|\|$_{N^{\prime}}$. But $\widetilde{F}_{N^{\prime}+1}$ is a function which has continuous derivatives of the $N^{\prime}$ first orders and $\square^{N^{\prime}+2} \widetilde{F}_{N^{\prime}+1}=\delta(x)$.

Define

$$
G(x)=\tilde{f}^{+} * \tilde{F}_{N^{\prime}+1}(x) .
$$

$G$ is a continuous function of $x$ and there exists a constant $K_{N^{\prime}}$ depending only of $N^{\prime}$ and such that

$$
|G(x)|=\left|f^{+} * \widetilde{F}_{N^{\prime}+1}(x)\right|<C K_{N^{\prime}}(1+\|x\|)^{9 N^{\prime}+8}
$$

and $f^{+}=\square^{N^{\prime}+2} G$, q.e.d.

The former demonstration was founded upon the fact that elements of $\mathscr{S}^{\prime}\left(\mathbb{R}_{4}\right)$ with supports in $\bar{V}+$ form an algebra for the convolution product. Distributions which have support contained in a set $A\left(A \subset \bar{V}^{+}\right)$, $A$ being such that $A+\bar{V}+\subset A$, form an ideal of this algebra, therefore $\operatorname{supp} G \subset A$.

We now apply this result to $f^{+}(p)$.

$$
\begin{aligned}
f^{+}(p) & =\left\langle\tilde{f}^{+}, e^{i(p, x)}\right\rangle=\left\langle\tau_{\alpha} \tilde{f}^{+}, e^{i(p, x-\alpha)}\right\rangle \\
& =\left\langle\tau_{\alpha} \tilde{f}^{+}, e^{i(p, x)}\right\rangle e^{-i p_{0} \alpha}, \quad p \in T^{+} .
\end{aligned}
$$


$\tau_{\alpha} f^{+}$is defined by

Thus

$$
\begin{aligned}
\left\langle\tau_{\alpha} \tilde{f}^{+}, \varphi\right\rangle & =\left\langle\tilde{f}^{+}, \tau_{-\alpha} \varphi\right\rangle \text { for any } \varphi \in \mathscr{S} \\
\left(\tau_{-\alpha} \varphi\right)(x) & =\varphi(x-\alpha) .
\end{aligned}
$$

$$
\operatorname{supp} \tau_{\alpha} f^{+} \subset A=\left\{\overline{\left.x \in \mathbb{R}_{4} \mid x \in \bar{V}^{+}, x_{0}>\alpha\right\}} \subset \bar{V}^{+}\right. \text {. }
$$

Obviously $A+\bar{V}+C A$ and therefore

$$
f^{+}(p)=\left(p^{2}\right)^{N^{\prime}+2} \int e^{i(p, x)} G(x) d^{4} x e^{-i p_{0} \alpha}
$$

with supp $G \subset A$.

From $|G(x)|<C^{\prime}(1+\|x\|)^{M}$ follows

$$
\begin{aligned}
\left|f^{+}(p)\right| & \leqq C^{\prime}\left|\left(p^{2}\right)^{N^{\prime}+2}\right| \int_{A}(1+\|x\|)^{M} e^{-(\operatorname{Im} p, x)} d^{4} x e^{\operatorname{Im} p_{0} \alpha} \\
& \leqq 2 \pi C^{\prime}\left|\left(p^{2}\right)^{N^{\prime}+2}\right| \int_{\alpha}^{\infty} d x_{0} \int_{0}^{x_{0}}(1+\|x\|)^{M} e^{-\operatorname{Im} p_{0} x_{0}} e^{|\operatorname{Im} \boldsymbol{p}| x} x^{2} d x e^{\operatorname{Im} p_{0} \alpha}
\end{aligned}
$$

and if we call $d\left(p, T^{+}\right)$or $d$ the distance of $p \in T^{+}$to the boundary of $T^{+}$then

$$
\left|f^{+}(p)\right|<C^{+}\left|\left(p^{2}\right)\right|^{N_{+}}\left(1+\frac{1}{d}\right)^{M_{+}} e^{|\operatorname{Im} \boldsymbol{p}| \alpha}, \quad p \in T^{+} .
$$

In the same way we can prove that

$$
\left|f^{-}(p)\right|<C^{-}\left|\left(p^{2}\right)\right|^{N_{-}}\left(1+\frac{1}{d}\right)^{M_{-}} e^{i \operatorname{Im} \boldsymbol{p} \mid \alpha}, \quad p \in T^{-}
$$

and, from the theorem of the Edge of the Wedge, we get:

$$
|f(p)|<C\left|\left(p^{2}\right)\right|^{N}\left(1+\frac{1}{d}\right)^{M} e^{\alpha|\operatorname{Im} \boldsymbol{p}|}, \quad p \in \omega
$$

where the constants $C, N$ and $M$ are the suprema of those which appear in the corresponding inequality $f^{+}$and $f^{-}$.

We now want to extend this majorization to the envelope of holomorphy $\Omega=H(\omega)$.

\section{Growth Properties of $\boldsymbol{f}$ in $\boldsymbol{\Omega}$}

We proceed in three stages. First we show that a function analytic in $\omega$, bounded there by $e^{\alpha|\operatorname{Im} \boldsymbol{p}|}$ keeps the same bound in $H(\omega)$. Then, by a suitable procedure of regularization, we suppress the polynomial growth at infinity in order to apply the result on the exponential bound.

\section{Functions Bounded Exponentially in a Domain of Jost-Lehman-Dyson}

We just state the theorem, the demonstration of which is found in Appendix I. 
Theorem I (BRos). Let $f(p)$ be an analytic function in $H\left(\mathscr{R} ; T^{+}, T^{-}\right)$ satisfying the inequality

$$
|f(p)|<C e^{\alpha|\operatorname{Im} \boldsymbol{p}|}, \quad p \in \omega
$$

then it satisfies in $H\left(\mathscr{R} ; T^{+}, T^{-}\right)$

$$
|f(p)|<C e^{\alpha|\operatorname{Im} p|} .
$$

\section{Elimination of the Polynomial Growth}

In inequality (2), we can replace $p^{2}$ by $\left(p-p^{\prime}\right)^{2}-m^{2}$, by changing the constant $C$ if necessary. According to the conditions [5] imposed to the region $\mathscr{R}$, there always exists a pair $\left(p^{\prime}, m\right)$ such that $\left[\left(p-p^{\prime}\right)^{2}-m^{2}\right]^{-1}$ is an analytic function of $p$ in $\mathscr{R} \cup T^{+} \cup T^{-}$.

We then define

$$
g(p)=f(p)\left[\left(p-p^{\prime}\right)^{2}-m^{2}\right]^{-N}
$$

$g(p)$ is analytic in $\mathscr{R} \cup T^{+} \cup T^{-}$and satisfis

$$
|g(p)|<C e^{\alpha|\operatorname{Im} p|}\left(1+\frac{1}{d}\right)^{M} .
$$

We now want to get an estimate of the growth of $g(p)$ in $H\left(\mathscr{R} ; T^{+}, T^{-}\right)$ after suppression of $(1+1 / d)^{M}$.

\section{Regularization and Deregularization Procedure}

The term $1 / d$ comes from the fact that $f(q)$ is not a $C^{\infty}$-function on the boundary of the domain of holomorphy. We can suppress this behaviour by smearing with test functions the boundary values of $f(p)$.

Let $\chi(r)$ belong to $C_{0}^{\infty}\left(\mathbb{R}^{+}\right)$, with $\operatorname{supp} \chi \in[0,1]$ and $\int_{0}^{1} r \chi(r) d r=1$. Then $\chi_{a}(r)=\chi(r / a), a>0$ satisfies $\int r \chi_{a}(r) d r=a^{2}$.

For $q \in \mathbb{C}_{4}, \varrho \in \mathbb{R}$ denote by $D(q ; \varrho)$ the polydisc $\left\{p \in \mathbb{C}_{4} \mid\|p-q\| \leqq \varrho\right\}$.

If $D(q, a)<\omega$, from the analyticity of $f(p)$ in $\omega$ we get for $r_{i}<a$, $i=0,1,2,3$

$$
\begin{aligned}
f(p)= & (2 \pi)^{-4} \int f\left(p_{0}+r_{0} e^{i \theta_{0}}, \ldots, p_{3}+r_{3} e^{i \theta_{3}}\right) d \theta_{0} \ldots d \theta_{3} \\
= & (a \sqrt{2 \pi})^{-8} \int f\left(p_{0}+r_{0} e^{i \theta_{0}}, \ldots, p_{3}+r_{3} e^{i \theta_{3}}\right) \chi_{a}\left(r_{0}\right) \ldots \chi_{a}\left(r_{3}\right) \\
& \cdot r_{0} d r_{0} d \theta_{0} \ldots r_{3} d r_{3} d \theta_{3}
\end{aligned}
$$

if then we set

$$
\begin{aligned}
-r_{j} e^{i \theta_{j}} & =\xi_{j}+i \eta_{j} \quad(j=0, \ldots, 3) \\
f(p) & =(a \sqrt{2 \pi})^{-8} \int f(p-\xi-i \eta) \prod_{j=0}^{3} \chi_{a}\left(\sqrt{\eta_{j}^{2}+\xi_{j}^{2}}\right) d \xi_{j} d \eta_{j} .
\end{aligned}
$$


Let us define now $T_{\varphi_{\eta}^{a}}(p)$ by

$$
T_{\varphi_{\eta}^{a}}(p)=\int \cdots \int_{-\sqrt{a^{2}-\eta_{j}^{2}}}^{+\sqrt{a^{2}-\eta_{j}^{2}}} f(p-\xi) \prod_{j=0}^{3} \chi_{a}\left(\sqrt{\eta_{j}^{2}+\xi_{j}^{2}}\right) d \xi_{j} .
$$

For every $a>0$, this formula defines $T_{\varphi_{\eta}^{a}}(p)$ as an analytic function of $p$ in $\widetilde{\mathscr{R}}_{a} \cup T^{+} \cup T^{-}$, where

with

$$
\mathscr{R}_{a}=\{q \in \mathscr{R} \mid C(q, a) \subset \mathscr{R}\}
$$

$$
C(q, a)=\left\{p \in \mathbb{R}_{4} \mid\|p-q\|<a\right\} .
$$

But $\tilde{\mathscr{R}}_{a} \cup T^{+} \cup T^{-}$is not a domain of holomorphy, therefore $T_{\varphi_{\eta}^{a}}(p)$ can be analytically continued in to the envelope of holomorphy $H\left(\tilde{\mathscr{R}}_{a} \cup T^{+} \cup T^{-}\right)$of $\widetilde{\mathscr{R}}_{a} \cup T^{+} \cup T^{-}$.

When $\operatorname{Im} p$ goes to zero in the tubes $T^{+}$and $T^{-}$, the convolution (3) defines $T_{\varphi_{\eta}^{a}}(p)$ as a $C^{\infty}$ function. It is the regularization of the tempered distributions $f^{+}$and $f^{-}$by the $C^{\infty}$ function $\varphi_{\eta}^{a}=\prod_{j} \chi_{a}\left(\sqrt{\eta_{j}^{2}}+\xi_{j}^{2}\right) \eta_{j}$ being fixed and $\left|\eta_{j}\right| \leqq a$.

We now introduce $F_{a}(p)$

$$
F_{a}(p)=a(\sqrt{2} \pi)^{-8} \int \cdots \int_{-\alpha}^{+\alpha} T_{\varphi_{\eta}^{a}}(p-i \eta) d \eta_{0}, \ldots, d \eta_{3}
$$

$F_{a}(p)$ is analytic in $\quad\left[H\left(\tilde{\mathscr{R}}_{a} \cup T^{+} \cup T^{-}\right)\right]_{a}$ and $f(p)=F_{a}(p)$ in $\left[H\left(\widetilde{\mathscr{R}}_{a} \cup T^{+} \cup T^{-}\right)\right]_{a}$.

We show in Appendix II that for domains of the Jost-Lehman-Dyson type, $\mathscr{R}$ being suffisantly "regular", there exists for a given $\varepsilon(\varepsilon>0)$, small enough, an integer $n$ independent of $\varepsilon$ such that

$$
\left[H\left(\tilde{\mathscr{R}} \cup T^{+} \cup T^{-}\right)\right]_{a^{1 / n}} \subset H\left(\tilde{\mathscr{R}}_{a} \cup T^{+} \cup T^{-}\right) \subset \Omega, \quad a<\varepsilon .
$$

In the following we will go to the limit $a=0$ in the different majorizations we will obtain. Therefore it is necessary that $\lim _{a \rightarrow 0} H\left(\widetilde{\mathscr{R}}_{a} \cup T^{+} \cup T^{-}\right)$ $=H\left(\tilde{\mathscr{R}} \cup T^{+} \cup T^{-}\right)$. This equality means that "taking the envelope of holomorphy" commutes in this case with taking the inductive limit or $\stackrel{\lim }{\longrightarrow} H\left(D_{v}\right)=H\left(\lim _{\longrightarrow} D_{v}\right)$. This result is obtained with the help of relation $\overrightarrow{(5)}$. In fact we have $H_{v} \subset H_{v}(D) \subset H(D)$ and the domains $H_{v}$ (here $\left.H_{v}=H_{a}=\left[H\left(\tilde{\mathscr{R}} \cup T^{+} \cup T^{-}\right)\right]_{a^{1 / n}}\right)$ form an increasing sequence approximating $H(D)$ from the inside. Therefore according to a well-known result in (FUKs [9]), $\lim _{\boldsymbol{v}} H_{v}=H(D)$ and the result, we need, follows.

${ }^{4}$ Let $K$ be an open set in $\mathbb{C}_{4},[K]_{a}$ means $[K]_{a}=\{q \in K \quad D(q, a) \subset K\}$. Obviously $[K]_{a} \subset K$. 
In Appendix III we show that $T_{\varphi_{\eta}^{a}}(p)$ is bounded by $M e^{\alpha \mid \operatorname{Im} \boldsymbol{p}} \mid / a^{m}$ in $\widetilde{\mathscr{R}}_{a} \cup T^{+} \cup T^{-}$, therefore, according to Theorem $\mathrm{I}$, it has the same bound in $H\left(\tilde{\mathscr{R}}_{a} \cup T^{+} \cup T^{-}\right)$, a being a fixed constant.

We extend this bound to $F_{a}(p)$, with the help of (4):

$$
\left|F_{a}(p)\right| \leqq M(\pi)^{-4} a^{-(m+4)} e^{\alpha|\operatorname{Im} \boldsymbol{p}-\boldsymbol{\theta}|} p \in\left[H\left(\tilde{\mathscr{R}}_{a} \cup T^{+} \cup T^{-}\right)\right]_{a}
$$

and $\theta=\left(\theta_{1}, \theta_{2}, \theta_{3}\right)$ such that $\left|\theta_{i}\right|<a$.

We now remove the $a$-dependence. Choose $\varepsilon$ small enough so that results of Appendix II can be applied. For any $p \in H\left(\tilde{\mathscr{R}} \cup T^{+} \cup T^{-}\right)$ there are two possible cases.

a) $d>\eta+\eta^{n}$.

$d$ is the distance $d\left(p,\left[H\left(\tilde{\mathscr{R}} C U T^{+} U T^{-}\right)\right)=d(p, \partial H)\right.$ and $\eta^{n}=\varepsilon$. Then according to the result of Appendix II we can find a number $a$, such that (Fig. 1).

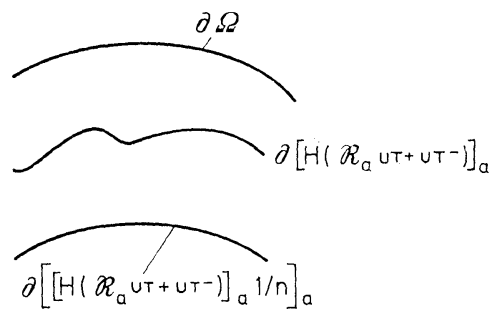

Fig. 1

$$
\left\{\begin{array}{l}
a<\varepsilon, \\
d<a+a^{1 / n}, \\
p \in\left[H\left(\tilde{\mathscr{R}}_{a} \cup T^{+} \cup T^{-}\right)\right]_{a} .
\end{array}\right.
$$

From $a^{1 / n}<\eta$ we get

$$
d(p, \partial H)<a^{1 / n}\left(1+a^{n-1 / n}\right)<a^{1 / n}\left(1+\eta^{n-1}\right)=C_{\eta}^{1 / n} a^{1 / n}
$$

and $\varepsilon$ being small enough, $C_{\eta}<2$ and $d^{n}<2 a$.

Therefore, from (6)

$$
\left|F_{a}(p)\right| \leqq M(\pi)^{-4} 2^{m+4} d^{-n(m+4)} e^{\alpha|\operatorname{Im} \boldsymbol{p}-\boldsymbol{\theta}|}
$$

or since $a<\varepsilon$, there exists $M^{\prime}$ independent of $a$ such that

b) $d>\eta+\eta^{n}$.

$$
\left|F_{a}(p)\right| \leqq M^{\prime} d-n(m+4) e^{\alpha|\operatorname{Im} \boldsymbol{p}|} .
$$

One can choose any $a<\eta^{n}=\varepsilon$ and according to (5),

$$
D\left(p, \eta^{n}\right) \subset H\left(\tilde{\mathscr{R}}_{a} \cup T^{+} \cup T^{-}\right),
$$

therefore $p \in\left[H\left(\tilde{\mathscr{R}}_{a} \cup T^{+} \cup T^{-}\right)\right]_{a}$ and the majorization (7) is valid with a constant $M^{\prime \prime}$ independent of $p$ for $d(p, \partial H)>\eta+\eta^{n}$. 
Finally for any $p$ in $\left[H\left(\tilde{\mathscr{R}}_{a} \cup T^{+} \cup T^{-}\right)\right]_{a}$ we have:

with $M$ independent of $\varepsilon$.

$$
\left|F_{a}(p)\right| \leqq M\left(1+\frac{1}{d}\right)^{n(m+4)} e^{\alpha \mid \operatorname{Im} \boldsymbol{p}_{i}}
$$

We deduce from this result that

$|f(p)| \leqq M\left(1+\frac{1}{d}\right)^{n(m+4)} e^{\alpha|\operatorname{Im} \boldsymbol{p}|}$ for $\quad p \in\left[H\left(\mathscr{R}_{a} \cup T^{+} \cup T^{-}\right)\right]_{a}$

since we have a bound which is independent of $a$, it is possible to pass to the limit and

$|f(p)| \leqq M\left(1+\frac{1}{d}\right)^{n(m+4)} e^{\alpha|\operatorname{Im} \boldsymbol{p}|}$ for $\quad p \in H\left(\tilde{\mathscr{R}} \cup T^{+} \cup T^{-}\right)$.

In collecting the various results we get

Theorem II. Let $f(p)$ be an analytic function of $p$ in $\tilde{\mathscr{R}} \cup T^{+} \cup T^{-}$ fullfilling the inequality

$$
|f(p)|<C e^{\alpha|\operatorname{Im} \boldsymbol{p}|}\left|\left(p^{2}\right)\right|^{N}\left(1+\frac{1}{d}\right)^{M}
$$

then, for $\mathscr{R}$ sufficiently regular, it fullfils in $H\left(\tilde{\mathscr{R}} \cup T^{+} \cup T^{-}\right)$

$$
|f(p)|<C^{\prime} e^{\alpha|\operatorname{Im} \boldsymbol{p}|}\left|\left(p^{2}\right)\right|^{N}\left(1+\frac{1}{d}\right)^{M^{\prime}} .
$$

$M^{\prime}$ being a constant which depends only of $M$ and of the regularity of $\mathscr{R}$.

\section{Conditions of Validity of Formula (1)}

We now give a set of properties for the Fourier transform of $\tilde{F}$ equivalent to the validity of formula (1). Our main tool will be the PaleyWiener-Schwartz-Hörmander [6] theorem which connects growth properties to support conditions.

Theorem. Let $K$ be a convex compact subset of $\mathbb{R}^{n}$. Let $H$ be the supporting function of $K$. Let f be a distribution with support in K. Then its Fourier Laplace transform obeys the following inequality:

a) $|f(p)| \leqq C(1+\|p\|)^{N} e^{H(\operatorname{Im} p)}, \quad p \in \mathbb{C}_{n}$ where $N$ is the order of f and $H(p)=\sup _{x \in K}(p, x)$.

Conversely every entire function analytic in $\mathbb{C}^{n}$ satisfying $(\alpha)$ is the Fourier-Laplace transform of a distribution with support in $K$.

Suppose now the validity of formula (1), the support in " $x$ " of $\widetilde{F}(x, \boldsymbol{t})$ being contained in $D(0)$, we can decompose $\widetilde{F}(x, \boldsymbol{t})$ in $\widetilde{F}^{ \pm}(x, \boldsymbol{t})[3,5]$ such that the support in " $x$ " of $\widetilde{F}^{+}(x, \boldsymbol{t})$ [resp. $\left.\tilde{F}^{-}(x, \boldsymbol{t})\right]$ is in $V^{+}$ (resp. $V^{-}$), the support in " $t$ " remaining unchanged, and:

$$
\widetilde{F}(x ; \boldsymbol{t})=\widetilde{F}^{+}(x ; \boldsymbol{t})-\widetilde{F}^{-}(x ; \boldsymbol{t}) .
$$

For $p$ belonging to $T^{+}$(or $T^{-}$) the action of $e^{i(p, x)} e^{-i(\boldsymbol{s}, \boldsymbol{t})}$ on $\widetilde{F}^{+}(x, \boldsymbol{t})$ [or $\left.\widetilde{F}^{-}(x, \boldsymbol{t})\right]$ is well defined and the result depends analytically on $p$ and $\boldsymbol{s}$. 
Hence, we define the analytic (entire in $s$ ) functions

$$
\begin{aligned}
& F^{+}(p, \boldsymbol{s})=\left\langle\widetilde{F}^{+}(x, \boldsymbol{t}), e^{i(p, x)} e^{-i(\boldsymbol{s}, \boldsymbol{t})}\right\rangle, \quad p \in T^{+}, \\
& F^{-}(p, \boldsymbol{s})=\left\langle\tilde{F}^{-}(x, \boldsymbol{t}), e^{i(p, x)} e^{-i(\boldsymbol{s}, \boldsymbol{t})}\right\rangle, \quad p \in T^{-} .
\end{aligned}
$$

According to condition iii) of the main theorem for any $\varphi(p) \in \mathscr{S}\left(\mathbb{R}_{4}\right)$ with supp $\varphi \subset \mathscr{R}$ and for any $\boldsymbol{s} \in \mathbb{C}_{3}$, one has:

$$
\left\langle\text { b.v. } F^{+}(p, \boldsymbol{s}), \varphi(p)\right\rangle=\left\langle\text { b.v. } F^{-}(p, \boldsymbol{s}), \varphi(p)\right\rangle
$$

therefore we can apply the Edge of the Wedge theorem for functions depending analytically on $\boldsymbol{s}$, and consequently there exists a function $F(p, \boldsymbol{s})$ analytic in $\Omega \times \mathbb{C}_{3}$ whose restrictions on $T^{+}$and $T^{-}$are respectively $F^{+}(p, \boldsymbol{s})$ and $F^{-}(p, \boldsymbol{s})$.

Now, from formula (1) we get

$$
\left.F(p, \boldsymbol{s})\right|_{\boldsymbol{s}=\boldsymbol{p}}=f(p), \quad p \in \Omega .
$$

According to the Paley-Wiener theorem conditions iv) implies:

for $(p, \boldsymbol{s}) \in \Omega \times \mathbb{C}_{3}$.

$$
|F(p, \boldsymbol{s})|<C(p)(1+\|\boldsymbol{s}\|)^{L} e^{\alpha|\operatorname{Im} s|}
$$

This inequality restricted to $\boldsymbol{s}=\boldsymbol{p}$ gives

$$
|f(p)|<C(p)(1+\|p\|)^{L} e^{\alpha|\operatorname{Im} p|} \quad \text { for } \quad p \in \Omega .
$$

This result is consistent with the growth properties, we directly found for $f$ (since $\left|p^{2}\right| \leqq\|p\|^{2}$ ) if $C(p)$ is polynomial in $p$.

We have to remark that the conditions imposed on $\tilde{F}(x ; \boldsymbol{t})$ in the main theorem do not in any case imply the unicity of the formula. In particular the orders (relative to $x$ or $\boldsymbol{t}$, or both) are not specified for the distribution $\widetilde{F}$.

Different types of growth can be proposed, which are compatible with (9) and (11). For instance ${ }^{5}$

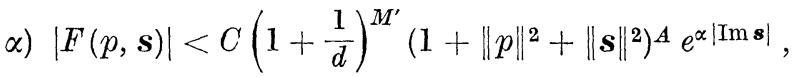
B) $|F(p, s)|<C\left(1+\frac{1}{d}\right)^{M^{\prime}}\left(1+\left\|p_{0}\right\|^{2}+\|\boldsymbol{s}\|^{2}\right)^{A} e^{\alpha|\operatorname{Im} s|}$,
$\gamma)|F(p, s)|<C\left(1+\frac{1}{d}\right)^{M^{\prime}}\left(1+\|p\|^{2}+\|\boldsymbol{p}-\boldsymbol{s}\|^{2}\right)^{A} e^{\alpha|\operatorname{Im} \boldsymbol{s}|}$,

the exponents $A$ are chosen in such a way they are compatible with (9).

Therefore from formula (1) we get the existence of a function of $p$ and $\boldsymbol{s}, F(p, \boldsymbol{s})$, analytic in $\Omega \times \mathbb{C}_{3}$, whose restriction to $\boldsymbol{s}=\boldsymbol{p}$ is $f(p)$ and which satisfies $\alpha$ ), $\beta$ ) or $\gamma$ ) with suitable exponents.

Conversely, if we know $F(p, s)$ satisfying the former properties, one can prove that there exists between $\tilde{f}(x)$ and $\widetilde{F}(x, \boldsymbol{t})$ [reconstructed from

${ }^{5}$ In these inequalities $d$ means either the distance to $C \Omega$ in $\mathbb{C}_{4}$ or the distance to $\mathrm{C}\left(\Omega \times \mathbb{C}_{3}\right)$ in $\mathbb{C}_{7}$, since they are equal. 
$F(p, s)]$ a relation of type (1). The important step, in this proof, is to recover the support in " $\boldsymbol{t}$ " by the help of the Paley-Wiener theorem.

This proves:

Theorem III. A sufficient condition for the validity of the main theorem for an $f$ as in Theorem $I I$ is the existence of a function $F(p, s)$ analytic in $\Omega \times \mathbb{C}_{3}$ and such that:

1) $\left.F(p, \boldsymbol{s})\right|_{\boldsymbol{s}=\boldsymbol{p}}=f(p)$,

2) $|F(p, \mathbf{s})| \leqq C\left(1+\frac{1}{d}\right)^{M^{\prime}}\left(1+\|p\|^{2}+\|\mathbf{s}\|^{2}\right)^{A} e^{\alpha|\operatorname{Im} s|}$.

$A$ being such that $A \geqq N$.

We have stated this theorem with the choice of a $\alpha$-type growth condition. We shall henceforth confine ourselves to this growth condition.

\section{Existence Theorem for $F(p, \vec{s})$}

At this stage we will use the technique of L. Hörmander following essentially the article: " $L$ ' estimates and existence theorems for the $\bar{\partial}$ operator" [8].

First we need a function $\varphi$ plurisubharmonic in $\Omega$ such that

$$
\int|f(p)|^{2} e^{-\varphi} d V_{\Omega}<+\infty
$$

with $d V_{\Omega}$ the Lebesgue measure on $\Omega$.

From the estimate (9)

$$
\int|f(p)|^{2} \frac{e^{-2 \alpha|\operatorname{Im} \boldsymbol{p}|}}{\left(1+\|p\| \|^{2}\right)^{2(N+n)}}\left(\frac{d}{1+d}\right)^{2 M^{\prime}} d V_{\Omega}
$$

is finite. We have added an extrapower $n$ to $\left(1+\|p\|^{2}\right)$ in order to insure the convergence at infinity (if $m=\operatorname{dim}_{\mathbb{C}} \Omega, 4 n \geqq m+\varepsilon(\varepsilon>0$ )).

This inequality implies that $f(p) \in L_{(0,0)}^{2}(\varphi, \Omega)^{6}$ with

$$
\begin{aligned}
\varphi(p)=2 \alpha|\operatorname{Im} \boldsymbol{p}| & +2 M^{\prime} \sup \left(-\log d+\log (1+\eta), \log \frac{1+\eta}{\eta}\right) \\
& +2(N+n) \log \left(1+\|p\|^{2}\right),
\end{aligned}
$$

$\eta$ being an arbitrary strictly positive number.

We have replaced $-\log \frac{d}{1+d}$ by $\sup \left(-\log d+\log (1+\eta), \log \frac{1+\eta}{\eta}\right)$ in order that plurisubharmonic functions appear. We show in Appendix IV that $\varphi(p)$ is plurisubharmonic.

We will show that there exists $F(p, s)$ analytic in $\Omega \times \mathbb{C}_{3}$ and which belongs to $L^{2}\left(\bar{\varphi}(p, \boldsymbol{s}), \Omega \times \mathbb{C}_{3}\right)$ with:

$$
\begin{aligned}
\bar{\varphi}(p, \boldsymbol{s})=2 \alpha|\operatorname{Im} \boldsymbol{s}| & +2 M^{\prime} \sup \left(-\log d+\log (1+\eta), \log \frac{1+\eta}{\eta}\right) \\
& +A \log \left(1+\|p\|^{2}+\|\boldsymbol{s}\|^{2}\right) .
\end{aligned}
$$

${ }^{6} f \in L_{(p, q)}^{2}(\varphi, \Omega)$ means $f$ is a differential form of type $(p, q)$ and $\varphi$ being a function locally bounded from above, measurable in $\Omega, \int|f(p)|^{2} e^{-\varphi} d V_{\Omega}$ exists. 
The coefficient $A$ will be specified later. We show in Appendix IV that $\bar{\varphi}$ is plurisubharmonic and that its lower bound of plurisubharmonicity is given by $e^{\chi}$ with:

$$
\chi=C^{t}-2 \log \left(1+\|p\|^{2}+\|s\|^{2}\right) .
$$

We can consequently apply Theorem 2.5.1 of Ref. [8] modified for our purpose.

Theorem IV. Let $\bar{\phi}$ be a plurisubharmonic function in $\Omega \times \mathbb{C}_{3}$ and $\varphi$ be a plurisubharmonic function in $\Omega, \Omega$ being a pseudoconvex open set in $\mathbb{C}_{3}$, such that

$$
|\bar{\varphi}(p, \boldsymbol{s})-\varphi(p)| \leqq C \quad \text { for } \quad|\boldsymbol{p}-\boldsymbol{s}| \leqq 1, \quad p \in \Omega, \quad \boldsymbol{s} \in \mathbb{C}_{3} .
$$

Then for any function $u$ analytic in $\Omega$ such that

$$
\int_{\Omega}|u|^{2} e^{-\varphi} d \sigma<+\infty
$$

$d \sigma$ where is the Lebesgue measure on $\Omega$, there exists an analytic function $U$ in $\Omega \times \mathbb{C}_{3}$ such that $U=u$ in $\Omega$ and

$$
\int_{\Omega \times \mathbb{C}_{3}}|U|^{2} e^{-\bar{\varphi}}\left(1+\|p\|^{2}+\|\boldsymbol{s}\|^{2}\right)^{-9} d V \leqq K \int_{\Omega}|u|^{2} e^{-\varphi} d \sigma
$$

$K$ being independent of $u$.

The difference between this theorem and that stated by HöRmaNDER lies in the fact that relation (14) is different and $\Omega$ is not the whole space. We sketch here a demonstration of Theorem IV.

$1^{\circ}$ ) Extension of $f(p)$ to $F_{1}\left(p, s_{1}\right)$ analytic in $\Omega \times \mathbb{C}_{1}$.

Let

with

$$
F_{1}\left(p, s_{1}\right)=f(p) \chi\left(p_{1}-s_{1}\right)-\left(p_{1}-s_{1}\right) \psi\left(p, s_{1}\right)
$$

such that

$$
\chi\left(p_{1}-s_{1}\right) \in C_{0}^{\infty}\left(\mathbb{R}^{+}\right)
$$

$$
\chi\left(p_{1}-s_{1}\right)=\chi\left(\left|p_{1}-s_{1}\right|\right)= \begin{cases}1 & \left|p_{1}-s_{1}\right|<1 / 2 \\ 0 & \left|p_{1}-s_{1}\right|>1\end{cases}
$$

To prove the existence of $F_{1}\left(p, s_{1}\right)$ we have to prove the existence of $\psi$. From the analyticity of $F_{1}$ it follows that

$$
\bar{\partial} F_{1}=\sum_{k} d \bar{z}_{k} \frac{\partial}{\partial \bar{z}_{k}} F_{1}=0
$$

where $z$ is a point in $\Omega \times \mathbb{C}_{1}$, i.e.

$$
\bar{\partial} F_{1}=f(p) \bar{\partial} \chi-\left(p_{1}-s_{1}\right) \bar{\partial} \psi=0
$$

which can be rewritten

$$
\bar{\partial} \psi=f(p) \frac{\bar{\partial} \chi}{p_{1}-s_{1}}=g .
$$

There exists such a $g$ since, when $\left|p_{1}-s_{1}\right|<1 / 2, \bar{\partial} \chi=0$. Thus, $g$ is a differential form of type $(0,1)$ satisfying $\bar{\partial} g=0$. We show now, that 17 Commun.math. Phys.,Vol.11 
there exists a form $\psi$ of type $(0,0)$ in $\Omega \times \mathbb{C}_{1}$ such that $\bar{\partial} \psi=g$. We need the following theorem (Hörmander, Ref. [8], Theorem 2.2.1').

Theorem V. Let $\Omega^{\prime}$ be a pseudo-convex open set of $\mathbb{C}_{n}$, let $\varphi$ be a plurisubharmonic function on $\Omega^{\prime}$ and $e^{\chi}, \chi \in C\left(\Omega^{\prime}\right)$, the lowest bound of plurisubharmonicity of $\varphi$. Then for any $g \in L_{(p, q)}^{2}\left(\Omega^{\prime}, \mathrm{loc}\right)^{7} q>0$ and such that

$$
\bar{\partial} g=0 \quad \int_{\Omega^{\prime}}|g|^{2} e^{-(\varphi+\chi)} d V_{\Omega^{\prime}},<+\infty
$$

one can find a form $\psi \in L_{p, q-1}^{2}\left(\Omega^{\prime}, \varphi\right)$ such that

$$
\bar{\partial} \psi=g \quad q \int_{\Omega^{\prime}}|\psi|^{2} e^{-\varphi} d V \leqq \int_{\Omega^{\prime}}|g|^{2} e^{-(\varphi+\chi)} d V .
$$

In our case we choose $\varphi$ and $\bar{\varphi}$ given by (12) and (13), with $A=2(N+n)$, then they satisfy relation (14) (see Appendix IV).

Define

$$
\varphi\left(p, s_{1}\right)=\left.\bar{\varphi}(p, \boldsymbol{s})\right|_{\begin{array}{l}
s_{2}=p_{2} \\
s_{3}=p_{3}
\end{array}}
$$

Now let us estimate $\int_{\Omega \times \mathbb{C}_{1}}|g|^{2} e^{-\varphi_{1}} d V_{\Omega \times \mathbb{C}_{1}}$

$$
\begin{aligned}
\int_{\Omega \times \mathbb{C}_{1}}|g|^{2} e^{-\varphi_{1}} d V & =\int_{\Omega} \int_{\left\|p_{1}-s_{1}\right\|<1}|g|^{2} e^{-\varphi_{1}} d V \\
& =\int_{\Omega} \int_{\left\|p_{1}-s_{1}\right\|<1}|g|^{2} e^{-\left(\varphi_{1}-\varphi\right)} e^{-\varphi} d V \\
& \leqq \pi e^{c}\left(2 c_{1}\right)^{2} \int_{\Omega}|f|^{2} e^{-\varphi} d V<+\infty
\end{aligned}
$$

with $c_{1}$, the upper bound of $\left|\frac{\partial \chi}{\partial \bar{s}_{1}}\right|$ in the unit disc. This result shows that $g \in L_{(0,1)}^{2}\left(\Omega \times \mathbb{C}_{1}, \varphi_{1}\right)$ with $\varphi_{1}$ strictly plurisubharmonic.

By choosing

with

$$
\tilde{\varphi}_{1}=\varphi_{1}-\chi
$$

$$
\chi=c_{1}-2 \log \left(1+\|p\|^{2}+\left\|s_{1}\right\|^{2}\right)
$$

we can apply Theorem $\mathrm{V}$ and, from it, we get the existence of $\psi\left(p, s_{\mathbf{1}}\right)$ $\in L_{(0,0)}^{2}\left(\Omega \times \mathbb{C}_{1}, \tilde{\varphi}_{1}\right)$ such that $\tilde{\partial} \psi=g$.

There exists consequently, an analytic function $F_{1}\left(p, s_{1}\right)$ in $\Omega \times \mathbb{C}_{1}$, which restricts to $f(p)$ on the hyperplane $s_{1}=p_{1}$ and satisfies

$$
\int_{\Omega \times \mathbb{C}_{1}}\left|F_{1}\left(p, s_{1}\right)\right|^{2} e^{-\varphi_{1}}\left(1+\|p\|^{2}+\left\|s_{1}\right\|^{2}\right)^{-3} d V<+\infty .
$$

$\left.2^{\circ}\right)$ We can make again the same construction with, now, $F_{1}\left(p, s_{1}\right)$ as initial function. Step by step we arrive to $F(p, s)$. Therefore we can state the of $\Omega$.

${ }^{7} L^{2}(\Omega$, loc $)$ is the space of functions square integrable on any compact subset 
Theorem. Let $f(p)$ be an analytic function in $\Omega$, domain of holomorphy in $\mathbb{C}_{4}$, satisfying

$$
|f(p)| \leqq C e^{\alpha|\operatorname{Im} \boldsymbol{p}|}\left(1+\|p\|^{2}\right)^{N}\left(1+\frac{1}{d}\right)^{M^{\prime}}, \quad p \in \Omega
$$

then there exists a function $F(p, \mathbf{s})$ analytic in $\Omega \times \mathbb{C}_{3}$ such that

1) $|F(p, \boldsymbol{s})|_{\mathbf{s}=\boldsymbol{p}}=f(p)$,

2) $\int|F(p, s)|^{2} e^{-\bar{\varphi}(p, s)}\left(1+\|p\|^{2}+\|s\|^{2}\right)^{-9} d V<+\infty$

with $\bar{\varphi}(p, s)=2 \alpha|\operatorname{Im} s|+2 M^{\prime} \sup \left(-\log d+\log (1+\eta), \log \frac{1+\eta}{\eta}\right)$ $+2(N+n) \log \left(1+\|p\|^{2}+\|s\|^{2}\right)$.

From inequality 2) we can deduce a majorization for $F$ in $\Omega \times \mathbb{C}_{3}$. From the positivity of the integrand it follows that

$$
\int_{\mathbb{C}_{s}}|F(p, s)|^{2} e^{-\bar{\varphi}(p, s)}\left(1+\|p\|^{2}+\|s\|^{2}\right)^{-9} d V<+\infty
$$

almost everywhere in $p$.

By CAUCHY's integral formula this implies that

$$
|F(p, s)| e^{-\frac{1}{2} \bar{\varphi}(p, s)}\left(1+\|p\|^{2}+\|\boldsymbol{s}\|^{2}\right)^{-9 / 2}
$$

is bounded for almost every $P$. But the continuity of $F$ and the semicontinuity of $\bar{\varphi}$ implies this for every $p$. Therefore we have

Theorem $\mathbf{V}^{\prime}$. Let $f(p)$ be an analytic function in $\Omega$ domain of holomorphy in $\mathbb{C}_{4}$ and satisfying

$$
|f(p)| \leqq C e^{\alpha|\operatorname{Im} \boldsymbol{p}|}\left(1+\|p\|^{2}\right)^{N}\left(1+\frac{1}{d}\right)^{M^{\prime}}, \quad p \in \Omega
$$

then there exists a function $F(p, \mathbf{s})$ analytic in $\Omega \times \mathbb{C}_{3}$ such that

1) $\left.F(p, \mathbf{s})\right|_{\mathbf{s}=\boldsymbol{p}}=f(p)$,

2) $|\boldsymbol{F}(p, \mathbf{s})|<C^{\prime} e^{\alpha|\operatorname{Im} \boldsymbol{s}|}\left(1+\frac{1}{d}\right)^{M^{\prime}}\left(1+\|p\|^{2}+\|\boldsymbol{s}\|^{2}\right)^{N+\frac{11}{2}+\varepsilon}$.

$\varepsilon$ being any positive number.

Acknowledgements. It is a pleasure to thank Pr. H. J. Borchers who introduced us to this problem and provided fruitful advice and Dr. J. LAscoux whose tenacity has compelled us to solve it.

We are particularly grateful to V. GLASER and J. Bros for valuable advice during the progress of our work.

Finally we also acknowledge useful discussions with R. STORA and J. Bessis.

\section{Appendix I}

In a preliminary version of this work, we needed a theorem of BRos concerning functions bounded by $e^{\alpha\left|\operatorname{Im} p_{0}\right|}$ in a domain of JosT-LEHMANDyson. We give here a proof for functions bounded by $e^{\alpha \mid \operatorname{Im} p}$ which is very close to the Bros' one. 
Theorem (Bros). Let $f(k)$ be a function analytic in $\Omega=H\left(\mathscr{R} ; T^{+}, T^{-}\right)^{8}$ which satisfies

$$
|f(k)|<C e^{\alpha|\boldsymbol{q}|} \quad k=p+i q \quad k \in \mathscr{R} \cup T^{+} \cup T^{-} .
$$

Then it satisfies in $\Omega$

$$
|f(k)| \leqq C e^{\alpha|\boldsymbol{q}|} .
$$

Define $F(w, k)=\left[C e^{-i w}-f(k)\right]^{-1}$ with $w=u+i v, C$ being the positive constant of inequality (1). $F(k, w)$ is analytic at any point $(w, k)$ such that $f(k)$ is analytic and $C e^{-i w}-f(k)$ different from zero. From the hypothesis, this last condition is fulfilled if

$$
\begin{gathered}
k \in \mathscr{R} \cup T^{+} \cup T^{-}, \\
\left|e^{-i w}\right| \geqq e^{\alpha|\boldsymbol{q}|} \quad \text { or } \quad v \geqq \alpha|\boldsymbol{q}| .
\end{gathered}
$$

Thus $F(w, k)$ is analytic, in particular, in a neighborhood of the union of the following three regions:

$$
\begin{array}{ll}
\text { the tube } T^{+}=\{(w, k)|v>\alpha| \boldsymbol{q} \mid, & \left.q \in V^{+}\right\} \\
\text {the tube } T^{-}=\{(w, k)|v>\alpha| \boldsymbol{q} \mid, & \left.q \in V^{-}\right\}
\end{array}
$$

the real region $\mathscr{R}_{1}=\{(w, k) \mid v=0, \quad q=0, \quad k=p \in \mathscr{R}\}$.

Consequently $F(w, k)$ is analytic in the envelope of holomorphy of $\tilde{\mathscr{R}}_{1} \cup T^{+} \cup T^{-}$, where $\tilde{\mathscr{R}}_{1}$ is the intersection of a complex neighborhood of $\mathscr{R}_{1}$ (in $\mathbb{C}_{5}$ ) with the convex envelope $T$ of $T^{+} \cup T^{-}$. We are going to show that the envelope of holomorphy of $\tilde{\mathscr{R}}_{1} \cup T^{+} \cup T^{-}$is:

$$
T \cap\{(w, k) \mid k \in \Omega\} .
$$

First, we restrict ourselves to the case where the $k$-space is 2 -dimensional, and prove the following lemma.

Lemma. We consider in the space $\mathbb{C}_{3}$ of variables $w=u+i v$, $\zeta_{1}=\xi_{1}+i \eta_{1}, \zeta_{2}=\xi_{2}+i \eta_{2}$ the tubes

$$
\begin{array}{lll}
T^{\prime+}=\left\{\left(w, \zeta_{1}, \zeta_{2}\right)|v>\alpha| \eta_{1}-\eta_{2} \mid,\right. & \eta_{1}>0, & \left.\eta_{2}>0\right\}, \\
T^{\prime \prime}=\left\{\left(w, \zeta_{1}, \zeta_{2}\right)|v>\alpha| \eta_{1}-\eta_{2} \mid,\right. & \eta_{1}<0, & \left.\eta_{2}<0\right\}
\end{array}
$$

and the region

$$
\mathscr{R}^{\prime}=\left\{\left(w, \zeta_{1}, \zeta_{2}\right) \mid v=\eta_{1}=\eta_{2}=0\left(\xi_{1}, \xi_{2}\right) \in \mathscr{R}\right\}
$$

where $\mathscr{R}$ is bounded with space-like curves and $h$-convex in the space $\left(\xi_{1}, \xi_{2}\right)$.

Let $T^{\prime}$ be the convex envelope of $T^{\prime \prime} \cup T^{\prime}-$ and $\Omega^{\prime}$ the domain of DysoN associated to $\mathscr{R}^{\prime}$ and to the tubes

$$
\left\{\left(\zeta_{1}, \zeta_{2}\right) \mid \eta_{1}>0, \eta_{2}>0\right\} \text { and }\left\{\left(\zeta_{1}, \zeta_{2}\right) \mid \eta_{1}<0, \eta_{2}<0\right\} \text {. }
$$

Then, the enveloppe of holomorphy of $T^{\prime+} \cup T^{\prime}-\cup \tilde{\mathscr{R}}^{\prime}$ is the intersection of $T^{\prime}$ with $\left\{\left(w, \zeta_{1}, \zeta_{2}\right) \mid\left(\zeta_{1}, \zeta_{2}\right) \in \Omega^{\prime}\right\}$.

${ }^{8}$ We remember that $\mathscr{R}$ is $h$-convex, which means that every branch of admissible hyperboloid (in Dyson's meaning) cuts $\mathscr{R}$ along a connected arc. Under these conditions $\mathscr{R}$ coincides with the set of all the real points of $\Omega$. 
Proof. $T^{\prime}$ is the union of $T^{\prime+}, T^{\prime}$ and the tubes

$$
\begin{aligned}
& T_{1}=\left\{\left(w, \zeta_{1}, \zeta_{2}\right)|v>\alpha| \eta_{1}-\eta_{2} \mid, \eta_{1}>0, \eta_{2}<0\right\}, \\
& T_{2}=\left\{\left(w, \zeta_{1}, \zeta_{2}\right)|v>\alpha| \eta_{1}-\eta_{2} \mid, \eta_{1}<0, \eta_{2}>0\right\} .
\end{aligned}
$$

$T_{1}$ can be looked at as the convex envelope of the union of the edge

and the face

$$
A=\left(\eta_{2}=0, \eta_{1}>0, v=\alpha\left|\eta_{1}-\eta_{2}\right|\right) \text { of } \bar{T}^{\prime+},
$$

$$
\Phi=\left(\eta_{2} \leqq 0, \eta_{1}>0, v \geqq \alpha\left|\eta_{1}-\eta_{2}\right|\right) \text { of } \bar{T}^{\prime-} .
$$

Consider, then, $A$ as belonging to the boundary of the tube

$$
\Theta=\left\{\left(w, \zeta_{1}, \zeta_{2}\right) \mid \eta_{2}>0, \eta_{1}>0, v^{\prime}=v-\alpha\left(\eta_{1}-\eta_{2}\right)<0\right\}
$$

and $\Phi$ as belonging to the boundary of the opposite tube. We are lead to calculate the envelope of holomorphy of $\Theta \cup-\Theta \cup \tilde{\mathscr{R}}^{\prime}$ and to restrict it to the tube $T_{1}$ (cf. Lemma 4, Ref. [10]).

This envelope of holomorphy is easily obtained by looking at $\mathscr{R}^{\prime}$ as a union of cubes

$$
\left\{a_{1}<\xi_{1}<b_{1}, a_{2}<\xi_{2}<b_{2}, \quad-c<u^{\prime}=u-\alpha\left(\xi_{1}-\xi_{2}\right)<0\right\} .
$$

Since $C$ can be taken arbitrarily large, independently of $a_{1}, a_{2}, b_{1}, b_{2}$, each of those contributions lead to a domain of holomorphy which is the product of the complex plane of the variable $w^{\prime}=u^{\prime}+i v^{\prime}$ by the domain of Dyson of the space $\left(\zeta_{1}, \zeta_{2}\right)$ relative to the region $\left\{a_{1}<\xi_{1}<b_{1}\right.$, $\left.a_{2}<\xi_{2}<b_{2}\right\}$ we consider. By taking the union over all the squares of this type contained in $\mathscr{R}^{\prime}$, we get, as it is well known, the domain of Dyson $\Omega^{\prime}$. Thus, the envelope of holomorphy of $\Theta \cup-\Theta \cup \tilde{\mathscr{R}}^{\prime}$ is $\left\{\left(w, \zeta_{1}, \zeta_{2}\right) \mid\left(\zeta_{1}, \zeta_{2}\right) \in \Omega^{\prime}\right\}$ and its restriction to $T_{1}$ belongs to the envelope of holomorphy of $T^{\prime}+\cup T^{\prime}-\cup \widetilde{\mathscr{R}^{\prime}}$.

The above results together with similar ones for $T_{2}$ complete the proof of the lemma.

Now, let us return to the four dimensional case.

Let $(w, k)$ belong to $T \cap\{(w, k) \mid k \in \Omega\}$. We want to show that this point belongs to the envelope of holomorphy of $T^{+} \cup T^{-} \cup \widetilde{\mathscr{R}}_{\mathbf{1}}$.

Since $k \in \Omega$, there exists a doubly inadmissible hyperbola which contains $k$; more, since the region $\mathscr{R}$ is assumed to be symetrical with respect to the plane $k_{0}=0$, this hyperbola $h$ can always be chosen in such a way that its plane is parallel to the $k_{0}$ axis (the reader is referred to the geometrical description of the domains of Dyson $[1,2])$. Let us cut, then, the figure by the three dimensional complex plane generated by the plane of this hyperbola and the $k_{0}$-axis. In this section of the five dimensional space, one can consider the section $T^{\prime+}, T^{\prime-}, \mathscr{R}^{\prime}$ of, respectively, the regions $T^{+}, T^{-}, \mathscr{R}_{1}$; by applying the lemma, one finds that the envelope of holomorphy of $T^{\prime+} \cup T^{\prime-} \cup \widetilde{\mathscr{R}}^{\prime}$ is $T^{\prime} \cap\left\{(w, k) \mid k \in \Omega^{\prime}\right\}$. The point 
$(w, k)$, we considered up to now, is by construction in $\Omega^{\prime}$, it remains for us to show that it is in $T^{\prime}$. If so, the point $(w, k)$ belongs to the envelope of holomorphy of $T^{\prime+} \cup T^{\prime} \cup \widetilde{\mathscr{R}}^{\prime}$ and therefore "a fortiori" to the $T^{+} \cup T^{-} \cup \widetilde{\mathscr{R}}_{1}$ ones.

Knowing that by hypothesis $(w, k) \in T$, one draws from $(v, q)$ the line parallel to the $q_{0}$ axis; one shows easily (Fig. 2) that it meets $T^{+}$and $T^{-}$ at two points $\left(v, q^{+}\right)$and $\left(v, q^{-}\right)$such that $q$ is on the segment $\left[q^{+}, q^{-}\right]$. Now, it is clear that the points $\left(w, p+i q^{+}\right)$and $\left(w, p+i q^{-}\right)$belong respectively to the tubes $T^{\prime+}$ and $T^{\prime \prime}$ (since by construction, these tubes are in a plane parallel to the $k_{0}$ axis).

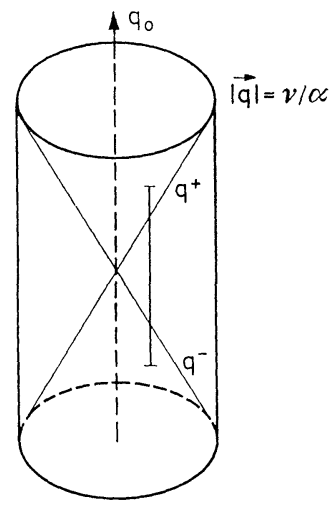

Fig. 2

Therefore we have proved that the function

$$
F(w, k)=\left[C e^{-i w}-f(k)\right]^{-1}
$$

is analytic for $k \in \Omega$ and $(w, k) \in T$.

This last condition can be written

$$
v>\alpha|\boldsymbol{q}|
$$

Thus for $k \in \Omega$, condition (3) implies:

$$
\left|C e^{-i w}\right|-|f(k)|=C e^{v}-|f(k)| \neq 0
$$

or

$$
|f(k)| \leqq C e^{\alpha|\boldsymbol{q}|} \quad \forall k \in \Omega .
$$

Remark. From the demonstration of the auxilliary lemma, it was obvious that the inequality is true in a 2-dimensional space, without any restriction to symmetrical region. This restriction appears, however, unavoidable in the four dimensional case. 


\section{Appendix II}

\section{$H\left(\mathscr{R} ; T^{+}, T^{-}\right)=\Omega$ and its "Translated" Parts}

\section{Two Dimensional Case}

Distance of the points (real or complex) on one hyperbola from a point on another one.

We assume that the centers of the hyperbolae are on the line $x_{0}=0$ and that the asymptotic directions make an angle of $45^{\circ}$ with the $x$ axis (Fig. 3).

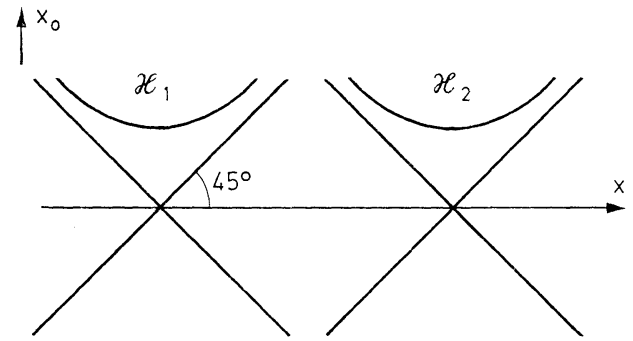

Fig. 3

Define

$$
\mathscr{H}_{1} \equiv x_{0}^{2}-x^{2}-m^{2}, \quad \mathscr{H}_{2} \equiv x_{0}^{2}-(x-u)^{2}-n^{2} .
$$

Let us take a point $P=P\left(x_{0}, x\right)$ on $\mathscr{H}_{1}$ and try to have an estimate of the distance $d\left(P, P^{\prime}\right)$ between $P$ and $P^{\prime} \in \mathscr{H}_{2}$. More precisely, we want the best possible majorization of $\inf _{P^{\prime} \in \mathscr{H}_{2}} d\left(P, P^{\prime}\right)$.

Let $(a, b)$ be the coordinates of $P$. Since one tries to majorize $\inf _{P^{\prime} \in \mathscr{H}_{2}} d\left(P, P^{\prime}\right)$ one can take for $P^{\prime}=P^{\prime}\left(\sqrt{b^{2}+u^{2}}, b+u\right)$.

Thus

$$
d^{2}\left(P, P^{\prime}\right)=u^{2}+\left(a-\sqrt{b^{2}+u^{2}}\right)^{2} \quad \text { with } \quad a=\sqrt{\overline{b^{2}+m^{2}}} .
$$

Finally

consequently

$$
\left(\sqrt{b^{2}+m^{2}}-\sqrt{b^{2}+n^{2}}\right)^{2} \leqq(m-n)^{2}
$$

hence

$$
d^{2}\left(P, P^{\prime}\right) \leqq u^{2}+(m-n)^{2},
$$

$$
\inf d^{2}\left(P, \mathscr{H}_{2}\right) \leqq u^{2}+(m-n)^{2} .
$$

For complex points, we have

$$
\begin{aligned}
& \mathscr{H}_{1} \Leftrightarrow\left\{\begin{array}{r}
x_{0}^{2}-y_{0}^{2}-x^{2}+y^{2}=m^{2} \\
x_{0} y_{0}=x y
\end{array}\right. \\
& \mathscr{H}_{2} \Leftrightarrow\left\{\begin{array}{r}
x_{0}^{2}-y_{0}^{2}-(x-u)^{2}+y^{2}=n^{2} \\
x_{0} y_{0}=y(x-u) .
\end{array}\right.
\end{aligned}
$$

(The $y$ 's are used for the imaginary parts.)

Let $P$ be the point $\left(x_{0}=a, x=b, y_{0}=c, y=d\right)$. The $a, b, c, d$ obey $a^{2}-c^{2}-b^{2}+d^{2}=m^{2}$ and $a c=b d$. 
A parametrization of points of $\mathscr{H}_{2}$ is given by:

$$
\begin{aligned}
x_{0} & =n \operatorname{ch} \varphi \cos \theta & & y_{0}=\varepsilon n \operatorname{sh} \varphi \sin \theta \\
x-u & =\varepsilon n \operatorname{sh} \varphi \cos \theta & & y=n \operatorname{ch} \varphi \sin \theta
\end{aligned} \quad \varepsilon^{2}=1 .
$$

Therefore one can set:

thus

$$
y_{0}=c, \quad x-u=b
$$

and

$$
x_{0}^{2}+y^{2}=n^{2}+c^{2}+b^{2}=a^{2}+d^{2}+u^{2}-m^{2}=A^{\prime 2}
$$

$$
a^{2}+d^{2}=m^{2}+c^{2}+b^{2}=A^{2} .
$$

Define

but

$$
\begin{aligned}
& a=A \cos \sigma, \quad x_{0}=A^{\prime} \cos \psi, \\
& d=A \sin \sigma, \quad y=A^{\prime} \sin \psi,
\end{aligned}
$$

therefore

$$
x_{0} c=y b
$$

and

$$
c / b=y / x_{0}=d / a=\operatorname{tg} \sigma=\operatorname{tg} \psi \quad \text { or } \quad \sigma=\psi
$$

$$
\begin{aligned}
d^{2}\left(P, P^{\prime}\right) & =\left(a-x_{0}\right)^{2}+(b-x)^{2}+\left(c-y_{0}\right)^{2}+(d-y)^{2} \\
& =\cos ^{2} \sigma\left(A-A^{\prime}\right)^{2}+u^{2}+\sin ^{2} \sigma\left(A-A^{\prime}\right)^{2} \\
& =u^{2}+\left(A-A^{\prime}\right)^{2}
\end{aligned}
$$

but

Thus

$$
\left(A-A^{\prime}\right)^{2}=\left(\sqrt{ } n^{2}+c^{2}+b^{2}-\sqrt{ } m^{2}+c^{2}+b^{2}\right)^{2} \leqq(m-n)^{2} .
$$

$$
\inf d^{2}\left(P, \mathscr{H}_{2}\right) \leqq u^{2}+(m-n)^{2}
$$

which shows that this formula is valid for real or complex points.

\section{Four Dimensional Case}

The boundary of $\Omega$ is given by the set of all the real or complex points obtained by cutting the hyperboloids bi-tangent to $\mathscr{R}$ (in our case $\mathscr{R}$, being symmetrical, the vertical of points of contact is parallel to the $x_{0}$-axis) with planes going through the points of contact. The boundary is therefore the union of all the hyperbolas cut on the hyperboloids by planes revolving around the vertical of points of contact [11].

We know that if $\mathscr{R}^{\prime} \subset \mathscr{R}$ then $\Omega^{\prime} \subset \Omega$. We have assumed that the boundary of $\mathscr{R}$ is made with space like surfaces, such that $\mathscr{R}$ is $h$-convex. $\mathscr{R}$ being symmetrical, if follows that the only cusp-like points (of order $q)^{9}$ of $\partial \mathscr{R}$ are in the plane $x_{0}=0$.

${ }^{9}$ We say that a point of a manifold is of order $q$ if every section, going through this point, by a 2-plane parallel to the $x_{0}$ axis is a curve which has a contact of order at least $q$ with its tangents at this point. 
We are going to show that each point of the boundary of $H\left(\tilde{\mathscr{R}}_{a} \cup T^{+} \cup T^{-}\right)$is within a distance, from the complementary of $\Omega$, less than " $a$ " raised to a specific power, and conversely.

First, let us go back for a moment to the two dimensional case. Let $\mathscr{C}$ and $\mathscr{C}^{\prime}$ be two curves which are mutually tangential at a point of order $q$. We suppose that in a neighborhood of this point they are smooth curves. We call $\mathscr{R}$ the region inside of the "angle" of contact (Fig. 4). Let $\mathscr{R}_{a}$ be the retracted region obtained with balls of radius $a(a$ is less than some $\varepsilon$ connected to the size of the neighborhood).

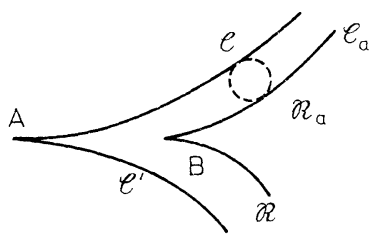

Fig. 4

Locally $\mathscr{R}$ is given by $|y|<x^{q}$ and $\mathscr{C}=y-x^{q} y \geqq 0$. Then

$$
\mathscr{C}_{a} \equiv\left\{x, y \mid y \geqq 0 \quad X=x+q \frac{2 a x^{q}}{\sqrt{1+q^{2} x^{2 q}}} Y=y-\frac{2 a}{\sqrt{1+q^{2} x^{2 q}}}\right\} .
$$

The point of retrogression of the "translated" curves is given by $Y=0$ $X \cong(2 a)^{1 / q}$.

Thus we have

$$
d\left(A, \mathscr{R}_{a}\right) \cong a^{1 / q} .
$$

Let us now return to the four dimensional case. Let $p$ be $a$ singular point on the boundary of $\mathscr{R}$. We assume that there exists an $\varepsilon$ such that at least one of the sections of $\mathscr{R}$, by 2 -planes passing through $p$, is regular everywhere else in a circle of radius $2 \varepsilon$, centered at $p$. That condition insures us that there is no nearby singularities in any directions. This is obviously the case when $\mathscr{R}$ is symmetrical, since singularities are only in the $x_{0}=0$ plane.

Consider then a section of $\partial \mathscr{R}$ (the boundary of $\mathscr{R}$ ) by a 2-plane $\pi$ parallel to the $x_{0}$-axis and look at the neighborhood of a regular point $A$. The hyperbola $\mathscr{H}_{A}$ is a boundary hyperbola tangent in $A$ to $\partial \mathscr{R}$. Its center is on the line $x_{0}=0$. We call $x$ the other axis in the 2-plane $\pi$. It is easy to see that if $\mathscr{H}_{A}=x_{0}^{2}-(x-u)-m^{2}$ (Fig. 5), then there exists in the same plane a hyperbola tangent to the section of $\partial \mathscr{R}_{a}$ and of equation $x_{0}^{2}-\left(x-u^{\prime}\right)^{2}-m^{\prime 2}$ with $u-u^{\prime} \in \mathcal{O}(a)$ and $m-m^{\prime} \in \mathcal{O}(a)$.

The distance is therefore majorized by $a^{2}$. We can convince ourselves of the uniformity of this majorization, that is to say, for any regular point of $\partial \mathscr{R}$, there exists a constant $C_{\mathscr{R}}$ such that $d^{2}\left(A, \partial \mathscr{R}_{a}\right) \leqq C_{\mathscr{R}} a^{2}$. 
Consider now in the plane $x_{0}=0$ the case of a singular point of order $n$. In one of the 2-planes, the extreme hyperbolae degenerate into cones

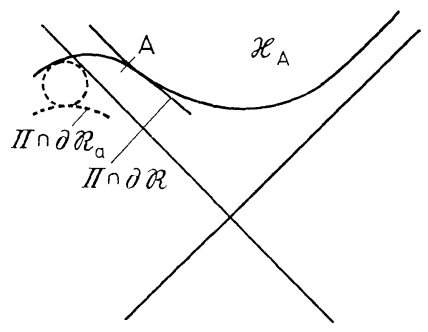

Fig. 5

of vertices $A$ and $B$. We thus get,

therefore

$$
d^{2}\left(A, \partial \mathscr{R}_{a}\right) \leqq C_{\mathscr{R}} a^{2 / n}, \quad d^{2}(B, \partial \mathscr{R}) \leqq C_{\mathscr{R}}^{\prime} a^{2}
$$

$$
H\left(\mathscr{R}_{a} ; T^{+}, T^{-}\right) \supset\left[H\left(\mathscr{R} ; T^{+}, T^{-}\right)\right]_{a^{1 / n}}
$$

since any point of the boundary of $H\left(\mathscr{R} ; T^{+}, T^{-}\right)$is at most at a distance of order $a^{1 / n}$ from the boundary of $H\left(\mathscr{R}_{a} ; T^{+}, T^{-}\right)$.

\section{Appendix III}

Majorization of $T_{\varphi_{\eta}^{a}}(p)$ in $\tilde{\mathscr{R}}_{a} \cup T^{+} \cup T^{-}$

$$
T_{\varphi_{\eta}^{a}}(p)=\int \cdots \int_{-\sqrt{a^{2}-\eta_{j}^{2}}}^{+\sqrt{a^{2}-\eta_{j}^{2}}} f(p-\xi) \prod_{j=0}^{3} \chi_{a}\left(\sqrt{\eta_{j}^{2}+\xi_{j}^{2}}\right) d \xi_{j}
$$

and

$$
\int_{0}^{1} r \chi_{a}(r) d r=a^{2}
$$

From the results we have obtained for $f(p)$ in the tubes

$$
\left|T_{\varphi_{\eta}^{a}}(p)\right|<M e^{\alpha|\operatorname{Im} \boldsymbol{p}|}\left\|\varphi_{\eta}^{a}\right\|_{m}
$$

with $m$ depending of $N$ (see formula 2) and $\tilde{\mathscr{R}}_{a} \cup T^{+} \cup T^{-}$

$$
\left\|\varphi_{\eta}^{a}\right\|_{m}=\sup _{\xi-\operatorname{Req} \in\left[-\sqrt{a^{2}-\eta^{2}}, \sqrt{a^{2}-\eta^{2}}\right]}\left|\sum_{i_{0}+i_{1}+i_{2}+i_{3} \leqq m} A_{i_{0} \ldots i_{3}} D_{\xi}^{i_{0}}+\cdots+i_{3} \prod_{j=0}^{3} \chi(j)\right|
$$

where $D_{\xi}^{i_{0}}+\cdots+i_{3}$ stands for

If we set

$$
\left(\frac{\partial}{\partial \xi_{0}}\right)^{i_{0}}\left(\frac{\partial}{\partial \xi_{1}}\right)^{i_{1}} \cdots\left(\frac{\partial}{\partial \xi_{3}}\right)^{i_{3}}
$$

then

$$
\frac{\xi_{j}-\operatorname{Re} q_{j}}{a}=u_{j}
$$

$$
\frac{\partial}{\partial \xi_{j}}=\frac{1}{a} \frac{\partial}{\partial u_{j}}
$$


but the derivatives of $\chi$ are bounded since $\chi$ is a $C^{\infty}$ function, therefore

$$
\left|\frac{\partial}{\partial \xi_{j}} \varphi_{\eta}^{a}\right|=\left|\prod_{i \neq j} \chi_{a}\left(\sqrt{\eta_{i}^{2}+\xi_{i}^{2}}\right) \frac{\partial}{\partial \xi_{j}} \chi_{a}\left(\sqrt{\eta_{j}^{2}+\xi_{j}^{2}}\right)\right| \leqq \frac{C}{a}
$$

and terms of $\left\|\varphi_{\eta}^{a}\right\|_{m}$ are of the form

$$
(1 / a)^{i_{0}+\cdots+i_{3}} C^{i_{0}+\cdots+i_{3}}
$$

Hence $\left\|\varphi_{\eta}^{a}\right\|_{m}<C_{m} \frac{1}{a^{m}}$ which is the majorization required.

\section{Appendix IV}

\section{Plurisubharmonicity of $\varphi$ and $\bar{\varphi}$}

We recall that $\varphi$ is plurisubharmonic in $\Omega$ (an open set in $\mathbb{C}_{n}$ ) if in the sense of distribution theory the quadratic form

$$
\sum_{j, k} t_{j} \bar{t}_{k} \frac{\partial^{2}}{\partial z_{j} \partial \bar{z}_{k}} \varphi(z)
$$

is positive definite for any $z$ in $\Omega$ and any $t=\left(t_{1}, \ldots, t_{n}\right)$ in $\mathbb{C}_{n}$. It will be strictly plurisubharmonic if there exsits a positive function $c(z) \in C(\Omega)$ such that

$$
\sum_{j, k} t_{j} \bar{t}_{k} \frac{\partial^{2}}{\partial z_{j} \partial \bar{z}_{k}} \varphi(z)-c(z) \sum_{j} t_{j} \bar{t}_{j}
$$

is positive definite for any $z$ in $\Omega$ and any $t$ in $\mathbb{C}_{n}$.

Since $\varphi$ and $\bar{\varphi}$ are the sum of three different types of terms, we have to check that each of those terms are plurisubharmonic.

1. $\Omega$ and $\Omega \times \mathbb{C}_{3}$ being domain of holomorphy $-\log d_{\Omega}=-\log d_{\Omega \times \mathbb{C}_{2}}$ is a plurisubharmonic function. A supremum of plurisubharmonic functions being subharmonic, $\sup \left(-\log d+\log (1+\eta), \log \frac{1+\eta}{\eta}\right)$ is plurisubharmonic.

2. $\log \left(1+\|s\|^{2}+\|p\|^{2}\right)$ or $\log \left(1+\|p\|^{2}\right)$ are strictly plurisubharmonic functions.

After some elementary calculation one gets :

$$
\sum t_{j} \bar{t}_{k} \frac{\partial^{2}}{\partial z_{j} \partial \bar{z}_{k}} \log \left(1+\|p\|^{2}+\|\boldsymbol{s}\|^{2}\right) \geqq \frac{\|t\|^{2}}{\left(1+\|p\|^{2}+\|\boldsymbol{s}\|^{2}\right)^{2}} \quad z=(p, \boldsymbol{s}) .
$$

3. $|\operatorname{Im} \boldsymbol{s}|$ or $|\operatorname{Im} \boldsymbol{p}|$ are strictly plurisubharmonic functions.. One obtains :

$$
\begin{aligned}
& \sum \frac{\partial^{2}}{\partial s_{j} \partial \bar{s}_{k}}|\operatorname{Im} \boldsymbol{s}| t_{j} \bar{t}_{k}=\theta(|\operatorname{Im} \boldsymbol{s}|) \frac{1}{|\operatorname{Im} \boldsymbol{s}|}\left(\|t\|^{2}-\|t . \operatorname{Im} \hat{\boldsymbol{s}}\|^{2}\right) \\
& +\|t\|^{2} \delta(|\operatorname{Im} \boldsymbol{s}|)+\|t \cdot \operatorname{Im} \hat{s}\|^{2}\left(\delta(|\operatorname{Im} \boldsymbol{s}|)+|\operatorname{Im} \boldsymbol{s}| \delta^{\prime}(|\operatorname{Im} \boldsymbol{s}|)\right) .
\end{aligned}
$$

Terms in $\delta$ and $\delta^{\prime}$ are positive on any positive test-function and $\|t\|^{2}-\|t . \operatorname{Im} \hat{\boldsymbol{s}}\|^{2} \geqq 0(\operatorname{Im} \hat{\boldsymbol{s}}$ is for a vector of direction $\operatorname{Im} \boldsymbol{s}$ and of norm 1$)$.

The denominator in $|\operatorname{Im} \boldsymbol{s}|$ is non singular since integrable in a three dimensional space. The last term vanishes identically. 
Therefore $\varphi$ and $\bar{\varphi}$ are strictly plurisubharmonic and if we define $\chi(z)$ by $c(z)=e^{\chi(z)}$

$$
\begin{aligned}
& \chi_{\varphi}(z)=\log 2(N+n)-2 \log \left(1+\|p\|^{2}\right), \\
& \chi_{\bar{\varphi}}(z)=\log A-2 \log \left(1+\|p\|^{2}+\|s\|^{2}\right) .
\end{aligned}
$$

Now we show that

$$
|\bar{\varphi}(p, \boldsymbol{s})-\varphi(p)| \leqq C \quad \text { for } \quad|\boldsymbol{p}-\boldsymbol{s}| \leqq 1 \quad p \in \Omega \quad \boldsymbol{s} \in \mathbb{C}_{3} .
$$

We give a bound for the three kind of terms previously considered

$\left.1^{\circ}\right) A=\|\operatorname{Im} \boldsymbol{p}|-| \operatorname{Im} \boldsymbol{s}\|$

$$
\begin{aligned}
A & =\left|\frac{\left.\operatorname{Im} \boldsymbol{p}\right|^{2}-|\operatorname{Im} \boldsymbol{s}|^{2}}{|\operatorname{Im} \boldsymbol{p}|+|\operatorname{Im} \boldsymbol{s}|}\right|=\left|\frac{\sum_{j}\left(\left|\operatorname{Im} p_{j}\right|^{2}-\left|\operatorname{Im} s_{j}\right|^{2}\right)}{|\operatorname{Im} \boldsymbol{p}|+|\operatorname{Im} \boldsymbol{s}|}\right| \\
& \leqq \sum_{i}\left(\frac{\left|\operatorname{Im} p_{i}\right|+\left|\operatorname{Im} s_{i}\right|}{|\operatorname{Im} \boldsymbol{p}|+|\operatorname{Im} \boldsymbol{s}|}\left(\left|\operatorname{Im} p_{i}\right|-\left|\operatorname{Im} s_{i}\right|\right)\right) \leqq 4 .
\end{aligned}
$$

$\left.2^{\circ}\right) B=\left|\log \left(1+\|p\|^{2}+\|s\|^{2}\right)-\log \left(1+\|p\|^{2}\right)\right|$

$$
\frac{1}{2} \leqq \frac{1+\|p\|^{2}}{1+\|p\|^{2}+\|s\|^{2}} \leqq 1 \text { thus } B \leqq \log 2 .
$$

$3^{\circ}$ ) Since the distance in $\Omega$ is equal to the distance in $\Omega \times \mathbb{C}_{3}$, the difference vanishes.

Therefore there exists a constant $C(>0)$ such that

$$
|\bar{\varphi}(p, \boldsymbol{s})-\varphi(p)|<C \text {. }
$$

\section{References}

0. Dyson, F. J.: Phys. Rev. 110, 579 and 110, 1460 (1958).

1. Omnes, R.: Contribution to: Relations de Dispersion et Particules Elementaires. DE WItT and Omnes, Eds. Paris: Hermann 1960.

2. Bros, J., A. Messiah, and R. Stora: J. Math. Phys. 2, 5, 639-651 (1961).

3. Schwartz, L.: Theorie des Distributions (2 Vol.). Paris: Hermann 1957-1959.

4. Streater, R. F., and A. S. Wightman: PCT, spin, statistics and all that. New-York: Benjamin 1964.

5. Vladimirov, V. S.: Methods of the theory of functions of several complex variables. Boston: MIT Press 1966.

6. Hörmander, L.: Supports and singular supports of convolution. Acta Math. 110, 3-4, 279-302 (1963).

7. Epstein, H.: Thesis (non published) and 1965 Brandeis University Summer Institute in Theoretical Physics, Vol. I.

8. Hörmander, L.: $L^{2}$ estimates and existence theorem for the $\bar{\partial}$ operator. Acta Math. 113, 91-152 (1965); or An introduction to complex analysis. In: Several variables. Princeton, N. J.: D. van Nostrand Comp. Inc. 1966.

9. Fuks, B. A.: Theory of analytic functions of several complex variables, Vol. 8 and Vol. 14 of Translations of Mathematical Monographes.

10. Bros, J., H. Epstein, and Glaser: Nuovo Cimento 31, 1265 (1964).

11. - C. Itzzykson, and F. Pham: Representations intégrales de fonctions analytiques et formule de Jost-Lehmann-Dyson. Ann. Inst. Poincaré 5, 1 (1966).

R. SÉNÉor

Centre de Physique Théorique

de l'Ecole Polytechnique

17, rue Descartes

F 75 Paris V 